\title{
Singular Potentials in Quantum Mechanics and Ambiguity in the Self-Adjoint Hamiltonian ${ }^{\star}$
}

Tamás FüLÖP

Montavid Research Group, Budapest, Soroksári út 38-40, 1095, Hungary

E-mail: tamas.fulop@gmail.com

Received August 07, 2007, in final form November 08, 2007; Published online November 16, 2007

Original article is available at http://www.emis.de/journals/SIGMA/2007/107/

\begin{abstract}
For a class of singular potentials, including the Coulomb potential (in three and less dimensions) and $V(x)=g / x^{2}$ with the coefficient $g$ in a certain range ( $x$ being a space coordinate in one or more dimensions), the corresponding Schrödinger operator is not automatically self-adjoint on its natural domain. Such operators admit more than one self-adjoint domain, and the spectrum and all physical consequences depend seriously on the self-adjoint version chosen. The article discusses how the self-adjoint domains can be identified in terms of a boundary condition for the asymptotic behaviour of the wave functions around the singularity, and what physical differences emerge for different selfadjoint versions of the Hamiltonian. The paper reviews and interprets known results, with the intention to provide a practical guide for all those interested in how to approach these ambiguous situations.
\end{abstract}

Key words: quantum mechanics; singular potential; self-adjointness; boundary condition

2000 Mathematics Subject Classification: 81Q10

\section{Introduction}

Let us consider a quantum mechanical Schrödinger Hamiltonian

$$
H=-\frac{\hbar^{2}}{2 m} \triangle+V
$$

where the real potential $V$ is singular, for example, $V(r) \sim \frac{1}{r}$ or $V(r) \sim \frac{1}{r^{2}}$. To keep the discussion technically simple, let our configuration space be a one dimensional interval or half line or line. Remarkably, the principles and methods to come will be valid for higher dimensional configuration spaces, too, it is only the amount of technicalities that increases with the dimensions. It is also important to note that the half line case plays a direct role in a number of higher dimensional problems as well. For instance, in a higher dimensional setting with a central symmetric potential that is divergent at the centre, one can perform a separation of variables in a spherical coordinate system, and the ambiguity that will be our topic here appears in the radial part of the system, which is actually a half line problem.

Now, in a straightforward manner, let us innocently search for all the eigenfunctions of this differential operator. Then, depending on our "luck", we may encounter some surprising difficulties ${ }^{1}$. Namely, we may find that, actually, there are "too many" eigenfunctions, in the sense that they are not mutually orthogonal, are not linearly independent, and form an overcomplete system rather than a basis in the Hilbert space of square integrable functions.

${ }^{\star}$ This paper is a contribution to the Proceedings of the 3-rd Microconference "Analytic and Algebraic Methods III". The full collection is available at http://www.emis.de/journals/SIGMA/Prague2007.html

${ }^{1}$ Naturally, this will not be a question of "luck", and we will see soon the mathematical criterion that tells that which potentials cause these difficulties. 
Moreover, we can observe that there are so many eigenfunctions that even the eigenvalues are not restricted to real values but any nonreal $\lambda \in \mathbb{C} \backslash \mathbb{R}$ also proves to be the eigenvalue of some (square integrable) eigensolution of the eigenvalue problem. Apparently, the self-adjointness of our, naively self-adjoint, Hamiltonian is seriously challenged. This can also be seen from that, for generic wave functions $\psi$ and $\chi$, applying integration by parts,

$$
(H \psi, \chi)-(\psi, H \chi)=\text { surface terms } \neq 0
$$

will be observable.

Now, if we don't have self-adjointness then we have no spectral theorem, no physical interpretation, and no unitary time evolution. Therefore, we are heavily motivated to restore self-adjointness, if only possible.

This last observation (2) may suggest us that the problem is created at the boundaries, especially at the location of singularity. We can try to cure the situation by requiring that all wave functions should vanish or decrease fast enough as we approach the singularity so that the surface terms tend to zero. Unfortunately, we will find that this way we lose all the eigenfunctions. Hence, this requirement cannot help in solving the problem. It seems that we need to keep some of the eigenfunctions and to dispose the rest of them.

Actually, the usual reaction to the problem - as many textbooks treat the three dimensional Coulomb problem, for example - is to declare that not all eigenfunctions are "acceptable". Suddenly, some extra ad hoc requirement is invented, which does not follow from the axioms/(general principles) of quantum theory but is introduced on the fly. Unfortunately, it is beyond the scope of this discussion to analyze those various conditions in detail. To give only a summary of the result of such a careful and honest analysis, one can reveal that

- those requirements are physically questionable,

- they have mathematically limited availability,

- different conditions can lead to different results,

- many consistent and valuable quantum mechanical models are lost by those requirements.

The situation is well illustrated, for example, by the paper [1], which, for the attractive Coulomb potential in one dimension, gives a critical overview of various existing treatments and their various different results - ands adds another new approach, which is also questionable both mathematically and physically.

However, we can follow another philosophy as well. Instead of the urge to choose, we can accept that the potential itself does not fix the model uniquely. When an application forces us to fix the ambiguity, some additional physical information will be needed. That information can come from experimental measurement or, if available, from some additional theoretical knowledge about the concrete case.

Therefore, for these potentials, let us search for all the quantum mechanically allowed cases (self-adjoint Hamiltonians) related to our initial Hamiltonian. Then, when in a concrete problem we need to choose one,

- knowing what possibilities exist may help to choose,

- if we have some additional information then it will be easier to utilize it,

- if measurement is needed to decide then we know what to measure and what to fit to the experimental data.

To carry out finding all the cases, it is advisable to review first what mathematics knows and tells about self-adjointness and about the possible ambiguity in it. 


\section{Self-adjoint extensions of symmetric operators}

We will make use of the following definitions and theorems ${ }^{2}$ (the validity of which is, nevertheless, not restricted to differential operators).

Let $A$ be a linear operator that is defined on a dense subset $\mathcal{D}(A)$ of a separable Hilbert space $\mathcal{H}$.

The adjoint $A^{+}$of $A$ is defined on those vectors $\chi \in \mathcal{H}$ for which there exists a $\tilde{\chi} \in \mathcal{H}$ such that

$$
(A \psi, \chi)=(\psi, \tilde{\chi}) \quad \text { for } \quad \forall \psi \in \mathcal{D}(A),
$$

and $A^{+}$is defined on such a $\chi$ as $A^{+} \chi:=\tilde{\chi}$.

$A$ is called symmetric if

$$
(A \psi, \chi)=(\psi, A \chi) \quad \text { for } \quad \forall \psi, \chi \in \mathcal{D}(A) .
$$

The adjoint of a symmetric $A$ is always an extension of it (i.e., $\mathcal{D}(A)$ is a subset of $\mathcal{D}\left(A^{+}\right)$, and $A$ and $A^{+}$act the same way on $\mathcal{D}(A)$ ).

$A$ is self-adjoint if $A=A^{+}$. (Which includes that their domains coincide.) $A$ is essentially self-adjoint if it admits a unique self-adjoint extension.

Remarkably, not the symmetric but only the self-adjoint operators are those for which the spectral theorem holds, and which are in a one-to-one correspondence with the strongly continuous one-parameter unitary groups $U(t)$ on $\mathcal{H}$ (to any $U(t)$, there is a unique self-adjoint $A$ such that $\left.U(t)=e^{-i A t}\right)$. Both these properties play an important role in the physical interpretation of quantum mechanics.

Next, from now on, let $A$ be a symmetric operator that is closed (for symmetric operators, this simply means the requirement $A^{++}=A$; any symmetric operator admits a closure - a minimal closed extension - which is actually nothing but its double adjoint). Let us also fix an arbitrary nonreal number $\lambda$.

The deficiency subspaces $\mathcal{E}_{\lambda}$ and $\mathcal{E}_{\lambda^{*}}$ are defined as the eigensubspace of $A^{+}$with respect to the eigenvalue $\lambda$ and the complex conjugate eigenvalue $\lambda^{*}$, respectively. The deficiency indices $n_{\lambda}$ and $n_{\lambda^{*}}$ are the dimension of $\mathcal{E}_{\lambda}$, resp. $\mathcal{E}_{\lambda^{*}} . A$ is self-adjoint if and only if $n_{\lambda}=n_{\lambda^{*}}=0$.

$A$ admits self-adjoint extensions if and only if its deficiency indices are equal ${ }^{3}, n_{\lambda}=n_{\lambda^{*}}=: n$. The self-adjoint extensions are in a one-to-one correspondence with the unitary maps from $\mathcal{E}_{\lambda}$ to $\mathcal{E}_{\lambda^{*}}$. Each unitary $U_{\mathrm{N}}: \mathcal{E}_{\lambda} \rightarrow \mathcal{E}_{\lambda^{*}}$ characterizes a self-adjoint extension $A_{U_{\mathrm{N}}}$ as the restriction of $A^{+}$to the domain

$$
\mathcal{D}\left(A_{U_{\mathrm{N}}}\right)=\left\{\psi_{0}+\psi_{\lambda}+U_{\mathrm{N}} \psi_{\lambda} \mid \psi_{0} \in \mathcal{D}(A), \psi_{\lambda} \in \mathcal{E}_{\lambda}\right\} .
$$

The unitary maps $U_{\mathrm{N}}$ act on an $n$ dimensional space so they can be parametrized by $n^{2}$ real parameters ${ }^{4}$.

After the characterization (3) given by von Neumann, let us see a more recent alternative description of the possible self-adjoint extensions, the so-called boundary value space approach:

If there exists an (auxiliary) Hilbert space $\mathcal{H}_{\mathrm{b}}$ (necessarily $n$ dimensional) and two linear maps $\Gamma_{1}, \Gamma_{2}: \mathcal{D}\left(A^{+}\right) \rightarrow \mathcal{H}_{\mathrm{b}}$ such that, for $\forall \psi, \chi \in \mathcal{D}\left(A^{+}\right)$,

$$
\left(A^{+} \psi, \chi\right)-\left(\psi, A^{+} \chi\right)=\left(\Gamma_{1} \psi, \Gamma_{2} \chi\right)_{\mathrm{b}}-\left(\Gamma_{2} \psi, \Gamma_{1} \chi\right)_{\mathrm{b}},
$$

\footnotetext{
${ }^{2}$ All mathematical ingredients quoted here and hereafter are taken from the sources $[2,3,4,5,6]$.

${ }^{3}$ For our differential operator Hamiltonian (1) with a real potential, this will always be the case, since such an operator is invariant under complex conjugation.

${ }^{4}$ For our differential operator Hamiltonian in one dimension, $n$ is finite (unless the potential admits infinitely many singularities). In higher dimensions, $n$ can be $\infty$, which happens when the singular locations are not finitely many points but lie, say, along a line.
} 
and to any two $\Psi_{1}, \Psi_{2} \in \mathcal{H}_{\mathrm{b}}$ there exists a $\psi \in \mathcal{D}\left(A^{+}\right)$satisfying

$$
\Gamma_{1} \psi=\Psi_{1}, \quad \Gamma_{2} \psi=\Psi_{2},
$$

then there is a one-to-one correspondence between the unitary maps $U \in \mathcal{U}\left(\mathcal{H}_{\mathrm{b}}\right)$ and the selfadjoint extensions of $A$, where a $U$ describes the domain of the corresponding $A_{U}$ as

$$
\mathcal{D}\left(A_{U}\right)=\left\{\psi \in \mathcal{D}\left(A^{+}\right) \mid\left(U-\mathbf{1}_{\mathrm{b}}\right) \Gamma_{1} \psi+i\left(U+\mathbf{1}_{\mathrm{b}}\right) \Gamma_{2} \psi=0\right\} .
$$

Heuristically, the second condition, related to (5), says that the auxiliary boundary Hilbert space $\mathcal{H}_{\mathrm{b}}$ must be a smallest one among those fulfilling the first condition, (4). If an $\mathcal{H}_{\mathrm{b}}$ is suitable for (4) then some appropriate trivial "enlargement", extension of it can also be suitable, e.g., by orthogonally adding some other Hilbert space to it, so this second condition is to ensure the efficiency of the description by removing the redundancy.

One can find that the former characterization of self-adjoint domains can be considered as a special case of the latter, with $\mathcal{H}_{\mathrm{b}}=\mathcal{E}_{\lambda}$. Another note to make is that, for a fixed $\mathcal{H}_{\mathrm{b}}$, the choice of appropriate $\Gamma$ s is not unique. Hence, it is not unique that which $U$ provides which self-adjoint version. Therefore, one should not - at least at the general level - attribute any distinguished meaning to that, having one choice of $\mathcal{H}_{\mathrm{b}}, \Gamma_{1}$ and $\Gamma_{2}$, which self-adjoint version is indexed by which unitary operator. (See more on it later.) A third remark is that, although the boundary value space approach may appear a rather abstract equipment at first sight, in applications we can find it simple, friendly and handy. Actually, historically it has been worked out to directly suit the special cases of differential operators where the "boundary values" (5) are indeed the limiting values of the wave function and its derivative at the boundary of the configuration space

- or appropriate combinations of them. Soon we will see examples that show how practical this approach is for differential operators.

\section{Finding the self-adjoint Hamiltonians}

Applying the content of the previous section to our differential operator of the type $-\frac{\hbar^{2}}{2 m} \triangle+V$, we first need to specify an initial domain on which it is symmetric. We can choose, for example, those wave functions $\psi$ which admit a continuous second derivative, vanish in a neighbourhood of any singularity of the potential and near any finite endpoints of our configuration space (if applicable), have compact support (so that

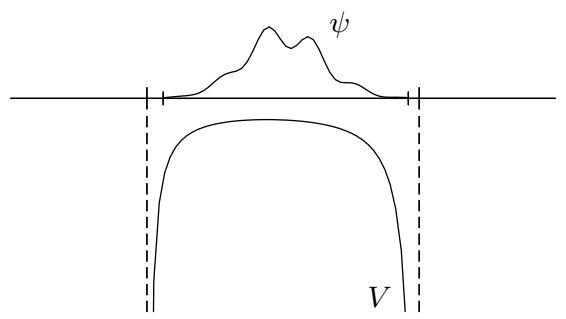
they also vanish "in a neighbourhood of infinity", for the case when the configuration space has $+\infty$ and/or $-\infty$ as "endpoint"), and $\psi, H \psi$ are square integrable. See the figure for an illustration. Such a domain is dense in $L^{2}$.

The closure of this symmetric operator will have a bit generalized domain, where the smoothness property is weaker ( $\psi$ and its derivative must be absolutely continuous), and the wave functions do not need to vanish identically around any "problematic" location but only to decrease fast enough so that the limiting surface terms of (2) emerging at the boundaries and singularities will be zero for any pairs $\psi, \chi$. At last, the adjoint of this closed operator will have the further generalized domain in which there is no restriction on the behaviour around the "problematic" locations.

Now, to specify the self-adjoint domains, which lie in between the symmetric domain and the adjoint domain, let us use the boundary value space description because that requires the least calculational efforts, and because there are known recipes how to find suitable candidates for the needed ingredients $\mathcal{H}_{\mathrm{b}}, \Gamma_{1}, \Gamma_{2}$. In what follows, we will see some instructive examples how the 
method works in practice. The first two examples are chosen to be simple to make the essence of the procedure apparent. In the meantime, they will already exhibit many of the general physical properties that typically arise when one has a singularity-induced or boundary-induced ambiguity in self-adjointness.

\section{Free particle on a half line}

As the first example, let us consider a free particle moving on a half line, which we wish to be bordered by a perfectly reflecting boundary to ensure the conservation of probability and, correspondingly, a self-adjoint domain for the Hamiltonian. (See the figure for an illustration and for the notations.)

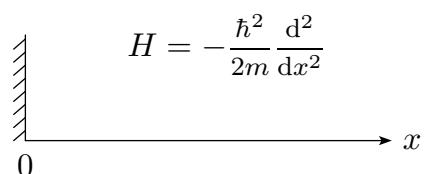
The most well-known way to reach this is to impose the Dirichlet boundary condition $\psi(0)=0$. However, other boundary conditions also provide self-adjoint possibilities, and physically it is a natural idea that there can be various different types of reflecting walls ${ }^{5}$. Therefore, exploring all the self-adjoint versions means to find the variety of perfectly reflecting walls that can be accounted for in the scope of this quantum mechanical model.

Starting with a symmetric domain and considering the corresponding adjoint domain as discussed in the previous section, integration by parts allows us to write, for the adjoint operator,

$$
\left(H^{+} \psi, \chi\right)-\left(\psi, H^{+} \chi\right)=-\frac{\hbar^{2}}{2 m}\left(\psi^{*} \chi^{\prime}-\psi^{* \prime} \chi\right)(+0)=-\frac{\hbar^{2}}{2 m} W\left[\psi^{*}, \chi\right](+0),
$$

where $W[\cdot, \cdot]$ denotes the Wronskian. In principle, we could expect a surface term related to $x \rightarrow \infty$ as well (since it is not immediately clear whether the square integrability of $\psi$ and $H^{+} \psi$ plus the degree of smoothness $\psi$ has provides a fast enough decrease towards infinity to send that term to zero): it will be explained in Section 6, at a general level, why it is absent indeed.

Comparing this formula with (4) shows that we can comply with the boundary value space approach with the choices

$$
\mathcal{H}_{\mathrm{b}}=\mathbb{C}, \quad \Gamma_{1} \psi=\psi(0), \quad \Gamma_{2} \psi=L_{0} \psi^{\prime}(0) .
$$

Here, we have introduced an auxiliary nonzero real length $L_{0}$, which does not play any principal role in the question of self-adjointness but is needed merely on dimensional grounds. Namely, the formalism requires $\Gamma_{1}$ and $\Gamma_{2}$ to have the same dimensions while $\psi$ and $\psi^{\prime}$ differ in a dimension of length ${ }^{6}$. Also, we have dropped the factor $-\frac{\hbar^{2}}{2 m}$ (that is, (4) has actually been fulfilled for $\left.A:=\frac{2 m L_{0}}{\hbar^{2}} H\right)$.

The other, "efficiency", condition needed for the boundary value space approach is also not hard to check. This point we are actually going to discuss later in Section 6, again at a general level.

Having fulfilled the requirements of the boundary value space method, we are allowed to harvest the fruit: the possible self-adjoint domains form a one-parameter family, indexed by $U \in \mathcal{U}(1)$, in a parametrized form, $U=e^{i \vartheta}, \vartheta \in[0,2 \pi)$, via the boundary condition

$$
\left(e^{i \vartheta}-1\right) \psi(0)+i\left(e^{i \vartheta}+1\right) L_{0} \psi^{\prime}(0)=0 .
$$

\footnotetext{
${ }^{5}$ We can imagine, for example, a long and thin nanowire, with a small piece of some material attached to its ends. Then, near one such end, quantum effects in the range of wavelengths that are much larger then the width of the wire as well as the size of this attached blob but smaller then the length of the wire may be well described by one of the "free particle on the half line" self-adjoint Hamiltonians, presumably different ones for different materials attached.

${ }^{6}$ For a full theory of how physical dimensions can be formulated and treated mathematically - via one dimensional vector spaces and their tensorial products, quotients and powers - see [7].
} 
We can rewrite this condition in the more simplified form

$$
\psi(0)+L \psi^{\prime}(0)=0, \quad L=L_{0} \cot \frac{\vartheta}{2} \in(-\infty, \infty) \cup\{\infty\}
$$

as well. Hence, the quantum mechanically allowed reflecting walls are characterized by an arbitrary real length parameter. The Dirichlet case corresponds to $L=0$, the Neumann boundary condition $\psi^{\prime}(0)=0$ is the case $L=\infty$, and the remaining cases, containing a nontrivial combination of the wave function and its derivative in the boundary condition, are often called the Robin boundary conditions.

It is instructive to see how the spectral properties, and correspondingly all physical properties, depend on the boundary parameter $L$. Omitting technical details, solving the eigenvalue problem for a given boundary condition yields $[8,9]$

$$
\begin{aligned}
& E>0: \quad \varphi_{k}(x)=\frac{1}{\sqrt{2 \pi}}\left(e^{-i k x}-e^{2 i \delta_{k}} e^{i k x}\right), \quad e^{2 i \delta_{k}}=\frac{1-i k L}{1+i k L}, \\
& E<0: \quad \varphi_{\text {bound }}(x)=\sqrt{\frac{2}{L}} e^{-x / L}, \quad E_{\text {bound }}=-\frac{\hbar^{2}}{2 m L^{2}} \quad(\text { only if } L>0) .
\end{aligned}
$$

Therefore, for example the existence and energy of a boundary bound state is heavily $L$ dependent. The $L$-dependence of the scattering phase shift can also be visualized by, e.g., the time delay, which is the difference between the time when the peak of an incoming wave packet reaches the wall and the time when the peak of the reflected packet leaves the wall. For an incoming packet concentrated in wave number around the value $-k$, the result is $[8,9]$

$$
\tau=\frac{-2 m L}{\hbar k\left(1+k^{2} L^{2}\right)}
$$

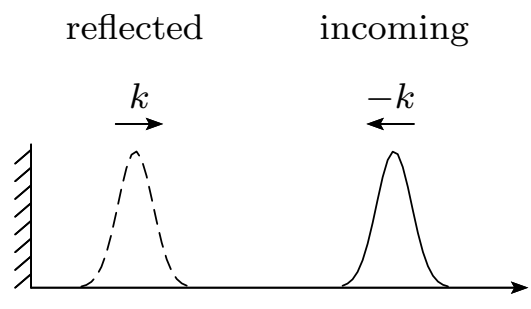

which seriously depends on $L$ (even its sign is decided by the sign of $L$ ). For further $L$-related effects and aspects, the Reader is asked to consult $[8,9]$.

\section{Free particle on a line with a point interaction}

In the next example, the particle can move on a line freely, except at one point where some object or disturbance or short-range potential-like effect resides

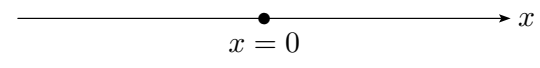
and can perform some nontrivial action on the particle. In this setting, the widely known example is the Dirac delta potential but the full family of possibilities again proves to be larger, and can be explored again via finding the possible selfadjoint versions of the initial Hamiltonian.

We can proceed similarly to the half line case, writing

$$
\left(H^{+} \psi, \chi\right)-\left(\psi, H^{+} \chi\right)=-\frac{\hbar^{2}}{2 m}\left\{\left(\psi^{*} \chi^{\prime}-\psi^{* \prime} \chi\right)(+0)-\left(\psi^{*} \chi^{\prime}-\psi^{* \prime} \chi\right)(-0)\right\},
$$

and finding agreement with (4) through

$$
\mathcal{H}_{\mathrm{b}}=\mathbb{C}^{2}, \quad \Gamma_{1} \psi=\left(\begin{array}{c}
\psi(+0) \\
\psi(-0)
\end{array}\right), \quad \Gamma_{2} \psi=L_{0}\left(\begin{array}{c}
\psi^{\prime}(+0) \\
-\psi^{\prime}(-0)
\end{array}\right) .
$$


Correspondingly, the family of self-adjoint possibilities can be indexed by $U \in \mathcal{U}(2)$, via

$$
\left(U-\mathbf{1}_{\mathbb{C}^{2}}\right) \Gamma_{1} \psi+i\left(U+\mathbf{1}_{\mathbb{C}^{2}}\right) \Gamma_{2} \psi=0 .
$$

Similarly to $L$ of the half line case, here, the four real parameters parametrizing the $U$ s can be chosen as two length scales $L_{+}, L_{-}$and two angles. Roughly speaking, the reason for two length parameters is that, now, the singular point has two sides. In parallel, one of the angles expresses the mixing between the left and the right side, and the other characterizes the strength of a short-range vector potential and thus causes a phase jump in the wave function at the singular point $^{7}$. In the case of zero mixing, which happens when the matrix $U$ is diagonal, there is no probability flow crossing the singularity, the two half lines physically decouple, and the system becomes a sum of two independent subsystems, two half line systems indeed (one with $L=L_{+}$ and the other with $L=L_{-}$).

Just as the sign of $L$ decides the number of bound states in the half line model, here the signs of $L_{+}$and $L_{-}$govern the number of bound states, which can thus be 0,1 or 2 . The scattering properties also depend on the boundary parameters, causing time delays, and making the point object to act as a low-pass or high-pass filter depending on the values of the parameters. See $[8,10,11,12]$ for further physical effects caused by the properties of the pointlike object.

\section{Singular potentials}

Now, we enter the situation having a potential that possesses not an abrupt ("hard") singularity like a Dirac delta but a continuously developing one like for the Coulomb potential ("soft" singularity). The complication with respect to the abrupt cases is that, here, the wave functions in the adjoint domain and their derivative are typically diverging when approaching the location of singularity. Therefore, we cannot choose such limits as boundary values. However, there exist some finite numbers hidden in the asymptotics of the wave functions, which can be extracted as follows. Let us first restrict ourselves to one side of the singularity.

One can start with observing ${ }^{8}$ that, for any $\psi, \chi$ in the adjoint domain, $W\left[\psi^{*}, \chi\right]$ has a finite limit when approaching the singularity. Next, fixing an arbitrary real value $E_{0}$, let us consider two real eigenfunctions $\varphi^{(1)}, \varphi^{(2)}$ of our Hamiltonian as a differential operator (i.e., omitting the requirements related to square integrability) for the eigenvalue $E_{0}$, two such solutions that satisfy $W\left[\varphi^{(1)}, \varphi^{(2)}\right]=1$. We will call them reference modes. Two possibilities exist:

1. Both reference modes are square integrable in a (one-sided) neighbourhood of the location of the singularity - this is called in the mathematical literature the limit-circle case $e^{9}$ (example: $\frac{\hbar^{2}}{2 m}\left(-\frac{\mathrm{d}^{2}}{\mathrm{~d} x^{2}}+\frac{5 / 16}{x^{2}}\right)$ on $\left.(0, \infty), E_{0}=0, \varphi^{(1)}(x)=x^{5 / 4}, \varphi^{(2)}(x)=-\frac{2}{3} x^{-1 / 4}\right)$,

2. At most, only a specific linear combination of them ${ }^{10}$ is square integrable around the singularity - this is called the limit-point case (example: $\frac{\hbar^{2}}{2 m}\left(-\frac{\mathrm{d}^{2}}{\mathrm{~d} x^{2}}+\frac{21 / 16}{x^{2}}\right)$, on $(0, \infty)$, $\left.E_{0}=0, \varphi^{(1)}(x)=x^{7 / 4}, \varphi^{(2)}(x)=-\frac{2}{5} x^{-3 / 4}\right)$.

It turns out that, if our problem is in the limit-point case then, for any two wave functions $\psi, \chi$ in the adjoint domain, $W\left[\psi^{*}, \chi\right]$ tends to zero at the singularity. Hence, a limit-point singularity does not create nonzero surface terms in (2) and, consequently, does not induce an ambiguity

\footnotetext{
${ }^{7}$ When replacing the line with a circle, this fourth parameter corresponds to the magnetic flux driven through the circle.

${ }^{8}$ For the mathematical results quoted in this Section, see, e.g., [3, 4, 5].

${ }^{9}$ The name has nothing to do with the geometry of our configuration space but is because of some historical and technical reasons.

${ }^{10} \mathrm{Up}$ to an overall complex factor, of course.
} 
in self-adjointness. As a special case, this is the reason why, in the "free particle on a half line" system, we did not need to worry about the surface terms towards infinity. Note that an infinite "endpoint" is always to be treated as a singular point, which can be seen, for example, from that the change of variable $x \rightarrow \frac{1}{x}$ brings it into finite (to zero), and via the accompanying unitary transformation $\tilde{\psi}(x):=\frac{1}{x} \psi\left(\frac{1}{x}\right)$ our symmetric differential operator is mapped into a one that is singular at zero (e.g., $-\frac{\hbar^{2}}{2 m} \frac{\mathrm{d}^{2}}{\mathrm{~d} x^{2}}$ is mapped to $-\frac{\hbar^{2}}{2 m}\left[\frac{\mathrm{d}}{\mathrm{d} x}\left(x^{4} \frac{\mathrm{d}}{\mathrm{d} x}\right)+2 x^{2}\right]$, and see the last paragraph of this Section).

In the limit-circle case where the surface terms do not vanish, what can be shown is that any $\psi$ in the adjoint domain is asymptotically similar to an appropriate linear combination of the reference modes, in the sense that

$$
\psi(x)=\left[c^{(1)}+\eta^{(1)}(x)\right] \varphi^{(1)}(x)+\left[c^{(2)}+\eta^{(2)}(x)\right] \varphi^{(2)}(x),
$$

where $\eta^{(1)}(x), \eta^{(2)}(x)$ vanish towards the singularity, and the limit numbers $c^{(1)}, c^{(2)}$ can be read off as the (always finite) limiting values of $-W\left[\varphi^{(2)}, \psi\right]$, respectively $W\left[\varphi^{(1)}, \psi\right]$, at the singularity. Furthermore, this asymptotic similarity proves to be strong enough for that, when we want to calculate the limit of a $W\left[\psi^{*}, \chi\right]$ at the singularity, we can replace $\psi(x)$ in it with $c^{(1)} \varphi^{(1)}(x)+c^{(2)} \varphi^{(2)}(x)$ (and $\chi$ can also be replaced with its similar approximation).

Adding

$$
W\left[\psi^{*}, \chi\right]=W\left[\varphi^{(1)}, \psi\right]^{*} W\left[\varphi^{(2)}, \chi\right]-W\left[\varphi^{(2)}, \psi\right]^{*} W\left[\varphi^{(1)}, \chi\right],
$$

which is nothing but a simple identity about determinants (recall $W\left[\varphi^{(1)}, \varphi^{(2)}\right]=1$ ), we find that the surface terms can be expressed in the desired form in terms of finite quantities so these limit numbers can be used for the purposes of $\Gamma_{1}, \Gamma_{2}$. The examples coming in the following sections will show this in close detail.

Now we can see the big practical advantage of the boundary value space method to using von Neumann's characterization directly. Namely, here we need to check square integrability of eigenfunctions only in a local neighbourhood of the singularity while, in the von Neumann approach, we need to do it over the whole configuration space. Besides, there are some additional benefits as well.

First, in the von Neumann method, we need to find eigenfunctions for a nonreal eigenvalue, while here for a real one. For example, many eigenequations become simpler for the eigenvalue $E_{0}=0$.

Second, we actually need only one solution, as the condition $W\left[\varphi^{(1)}, \varphi^{(2)}\right]=1$ allows us to find another one in a form

$$
\varphi^{(2)}(x):=\varphi^{(1)}(x) \int_{x_{0}}^{x} \frac{\mathrm{d} x}{\left[\varphi^{(1)}(x)\right]^{2}} .
$$

Third, we need the reference modes only approximately, to only such a preciseness that the limit of their Wronskian with any $\psi$ can be determined, plus that their local square integrability can be checked. In practice, this usually means that we need to know their leading and first subleading asymptotic behaviour.

In case our singularity is a so-called regular singular point then the asymptotic solutions are in fact known from the Frobenius method. It is usually advantageous to choose the regular solution for one of the reference modes.

As a last virtue to mention, it is also apparent that the need for a boundary condition, as well as its form, is decided only locally around the singularity and not globally along the whole configuration space. Using the von Neumann approach with the deficiency eigenfunctions this point would also remain hidden. Actually, if we are on an interval bordered by two limit-circle singular endpoints then we are allowed to use different reference modes at the two endpoints. 
Concerning the efficiency condition not yet discussed, the mathematical literature ensures that this will also be fulfilled with the $\Gamma_{1}, \Gamma_{2}$ chosen above. Indeed, it can be shown that, to any two complex numbers $c^{(1)}, c^{(2)}$, one can find a $\psi$ in the adjoint domain whose limit numbers are these $c^{(1)}$ and $c^{(2)}$. Observing that a regular endpoint (where the potential does not diverge) behaves the same way as a limit-circle singular endpoint from all relevant aspects - we can use $\chi^{(1)}(x)=-x$ and $\chi^{(2)}(x)=1$ as approximate reference modes - one can obtain that, in the special case of a regular endpoint, the limits of $\psi$ and $\psi^{\prime}$ as boundary values do satisfy the efficiency condition.

The presented description of the self-adjoint domains via reference modes contains some arbitrariness. One such freedom is in the value of $E_{0}$. Since the term containing $E_{0}$ in the eigenequation is overwhelmed by the diverging potential term, it is plausible to expect and can actually be proven that the asymptotic behaviour of the reference modes that decides the limit numbers of the wave functions does not depend on $E_{0}$. The other arbitrariness is how the two independent reference modes are chosen for a given $E_{0}$. This latter uncertainty, which is an $S L(2, \mathbb{R})$ amount of freedom, really influences the characterization. (As a special case of what has been mentioned about the non-uniqueness of $\Gamma$ s in Section 2.) The family of self-adjoint domains is nevertheless the same, what changes is only that which domain is indexed by which $U$. Correspondingly, parameters chosen to parametrize $U \in \mathcal{U}(2)$ may also change. Naturally, it is advantageous if we are able to introduce parameters in such a - reference mode-dependent way that they remain the same under changing the reference modes.

A last remark is that, should our Hamiltonian have a kinetic term with $\frac{\mathrm{d}}{\mathrm{d} x}\left(p(x) \frac{\mathrm{d} \psi}{\mathrm{d} x}\right)$ instead of $\frac{\mathrm{d}^{2} \psi}{\mathrm{d} x^{2}}$, we only need to replace $\frac{\mathrm{d} \psi}{\mathrm{d} x}$ with $p(x) \frac{\mathrm{d} \psi}{\mathrm{d} x}$ in the above formulas and considerations. Any possible zeros of $p(x)$ are also to be considered singularities which, with this replacement, can be treated analogously to the singularities of an operator with constant $p$. An example is provided by the operator $-\frac{\hbar^{2}}{2 m}\left[\frac{\mathrm{d}}{\mathrm{d} x}\left(x^{4} \frac{\mathrm{d}}{\mathrm{d} x}\right)+2 x^{2}\right]$ mentioned above. The zero of $p(x)=x^{4}, x=0$, indicates a singular point there. The reference modes are to be normalized to $p W\left[\varphi^{(1)}, \varphi^{(2)}\right]=1$, and can be chosen for $E_{0}=0$ as $\varphi^{(1)}(x)=x^{-1}, \varphi^{(2)}(x)=-x^{-2}$. These reference modes indeed behave singularly at $x=0$, and show that, in this case, the singularity is of limit-point type as neither of them is square integrable in a finite neighbourhood of $x=0$.

\section{Singular potential on a half line}

Let us again take two examples - actually, the generalizations of our previous two examples to apply the knowledge collected in the previous section.

First, let us consider a half line again, but now with a potential that diverges in a limit-circle way at the finite endpoint. Based on the findings and notations introduced in Sections 4 and 6, we do not need much explanation why the boundary value space approach and the corresponding characterization of self-adjoint domains can be given as ${ }^{11}$

$$
\mathcal{H}_{\mathrm{b}}=\mathbb{C}, \quad \Gamma_{1} \psi=W\left[\varphi^{(1)}, \psi\right](+0), \quad \Gamma_{2} \psi=W\left[\varphi^{(2)}, \psi\right](+0) ; \quad \Gamma_{1} \psi+L \Gamma_{2} \psi=0 .
$$

To be concrete, we may choose the three dimensional Coulomb problem, with the Hamiltonian

$$
H=\frac{\hbar^{2}}{2 m}\left(-\triangle+\frac{g}{r}\right)
$$

\footnotetext{
${ }^{11} \mathrm{An} L_{0}$ may be needed to introduce here or there, because of the already mentioned dimensional reason, but it is also possible that a coefficient from the potential can take this role. $L$ in $(7)$ may also have a dimension different than length, depending on the conventions we choose.
} 
After the usual separation of variables in spherical coordinates (including the radial mapping $\left.L^{2}\left((0, \infty), r^{2} \mathrm{~d} r\right) \rightarrow L^{2}((0, \infty), \mathrm{d} r)\right)$, the radial part,

$$
H_{\mathrm{rad}}^{(l)} \psi_{\mathrm{rad}}(r)=\frac{\hbar^{2}}{2 m}\left(-\frac{\mathrm{d}^{2}}{\mathrm{~d} r^{2}}+\frac{l(l+1)}{r^{2}}+\frac{g}{r}\right) \psi_{\mathrm{rad}}(r)
$$

will have an ambiguity in self-adjointness in the $l=0$ angular momentum channel because the centre is a limit-point singularity for the radial Hamiltonians $H_{\mathrm{rad}}^{(l)}$ for $l>0$ but is a limit-circle one for $l=0$.

For $l=0$, a satisfactory approximation for reference modes near $r=0$ is

$$
\varphi^{(1)}(r) \approx-r, \quad \varphi^{(2)}(r) \approx 1+g r \ln |g| r .
$$

These can be used in the boundary condition (7). For $L=0$, the widely known and usually considered case yields, in which the $\psi$ s allowed by the boundary condition are regular at the centre. In the other cases $L \neq 0$, a singular component is present in $\psi$ so $\psi^{\prime}$ diverges at the centre. Experiments make us quite confident in that, when the potential is intended to express a purely electromagnetic interaction, we should choose $L=0$ in addition. However, for a physical situation where some additional, short-range, interaction is expected to be present between the centre and the particle, a model with $L \neq 0$ is a good candidate. Mesic atoms, in which not electrons but some negatively charged mesons like $\pi^{-}$move around the proton-containing centre, provide an example for this, because of the short-range strong force also acting between the protons and mesons.

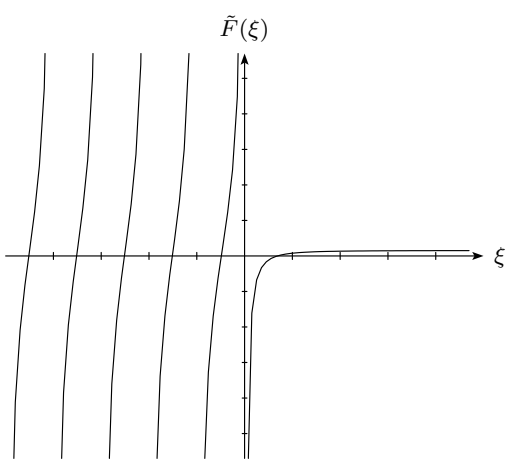

For generic $L$, the condition for bound states can be found [13] to be

$$
g \tilde{F}\left(\frac{g}{2 \sqrt{-2 m E / \hbar^{2}}}\right)=-\frac{1}{L}
$$

with

$$
\tilde{F}(\xi)=\Psi(1+\xi)-\ln |\xi|-\frac{1}{2 \xi}-\Psi(1)-\Psi(2),
$$

where $\Psi$ denotes the di-Gamma function.

If our potential is attractive, $g<0$, then, for $L=0$, we obtain the well-known bound state energies

$$
E_{n}=-\frac{R}{n^{2}}, \quad n=1,2, \ldots \quad(L=0) .
$$

However, taking for example the case $L=\infty$, the solutions of the transcendental equation (9) give a different infinite sequence of bound states in the $l=0$ angular momentum channel:

$$
E_{n}=-\frac{R}{\left(n-c_{n}\right)^{2}}, \quad n=1,2, \ldots \quad(L=\infty)
$$

with

$$
c_{1} \approx 0.5130, \quad c_{2} \approx 0.4879, \quad c_{3} \approx 0.4857, \quad \ldots, \quad c_{\infty} \approx 0.4844 \text { (limit). }
$$

The scattering properties - like the scattering length and phase shift quantities - and other physical aspects are similarly considerably influenced by the value of the self-adjointness parameter $L$. 


\section{Singular potential on a line}

Next, let us again extend our configuration space to a line, on which the potential admits a singularity that is of limitcircle type from both directions. Similarly to the "free line with a pointlike singular object" example seen in Section 4, the two sides of the singularity double the dimension of the boundary value space, and we also need to double (to extend) the reference modes. Having seen (6) and (7), no wonder that we choose

$$
\mathcal{H}_{\mathrm{b}}=\mathbb{C}^{2}, \quad \Gamma_{1} \psi=\left(\begin{array}{c}
W\left[\varphi^{(1)}, \psi\right](+0) \\
W\left[\varphi^{(1)}, \psi\right](-0)
\end{array}\right), \quad \Gamma_{2} \psi=\left(\begin{array}{c}
W\left[\varphi^{(2)}, \psi\right](+0) \\
-W\left[\varphi^{(2)}, \psi\right](-0)
\end{array}\right)
$$

and obtain the boundary conditions, with $U \in \mathcal{U}(2)$, as

$$
\left(U-\mathbf{1}_{\mathbb{C}^{2}}\right) \Gamma_{1} \psi+i\left(U+\mathbf{1}_{\mathbb{C}^{2}}\right) \Gamma_{2} \psi=0 .
$$

Here again, a certain subfamily of the family of self-adjoint Hamiltonians contains the separated cases where the system decouples to two independent half line subsystems. This means that, in those cases, the particle bounces back from the singularity. This phenomenon is present for attractive potentials as well, which is surprising for the physical intuition. It creates a feeling that, in those cases, something repulsive must happen at the location where the potential diverges.

On the other side, in the nonseparated cases we can observe reflection-transition, time delay and filter phenomena similarly to the case of a pointlike interaction on a line. This includes that we find tunneling through a repulsive potential as well [14], which is much more surprising

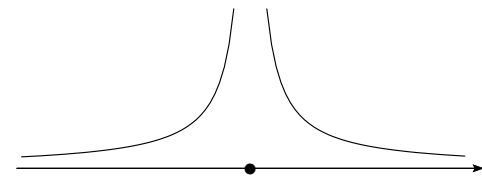
for such a "fat" infinite potential barrier than for a "thin" pointlike object. Again, one may try to illustrate these cases physically by that some attractive effect is present at the location where the potential is undefined.

Many other physical properties, like integrability for Calogero-type potentials whose singular term $\frac{g}{r^{2}}$ has $g<\frac{3 \hbar^{2}}{8 m}[15]$, are also dependent on the self-adjoint version chosen ${ }^{12}$. These dependences might be utilized in the future to build quantum devices like quantum gates, filters and qubits when the boundary parameters become tunable and controllable [16].

\section{Conclusion}

We have seen how the ambiguity in the self-adjointness of a Schrödinger Hamiltonian with a singular potential can be understood, and how it can be technically treated in a framework that requires only a reduced amount of calculational efforts.

Naturally, what has been presented here is only a narrow and practice-oriented extract from the extensive research field of boundary conditions and singular potentials and, more generally, of self-adjoint extensions. For further reading, in addition to the works already referred to, one can start with consulting, for example, [17, 18, 19, 20, 21, 22] and references therein.

To close the discussion with the message: the self-adjointness ambiguity caused by irregularities of the potential can be interpreted such that the system carries extra physical properties which can not be expressed via the potential function but through a boundary condition at each irregularity. These properties are to be fixed either by some additional theoretical knowledge or physical information about the system, or by measurement, fitting the unknown boundary parameters to experimental data.

\footnotetext{
${ }^{12}$ Writing $\frac{g}{r^{2}}$ as $\frac{\hbar^{2}}{2 m} \frac{l(l+1)}{r^{2}}$, ambiguity in the self-adjointness occurs for $l<\frac{1}{2}$. In parallel, the Hamiltonian will be bounded from below when $g \geq-\frac{\hbar^{2}}{8 m}$ (when $l$ is real).
} 


\section{Acknowledgements}

Work supported in part by the Czech Ministry of Education, Youth and Sports within the project LC06002.

\section{References}

[1] Gordeyev A.N., Chhajlany S.C., One-dimensional hydrogen atom: a singular potential in quantum mechanics, J. Phys. A: Math. Gen. 30 (1997), 6893-6909.

[2] Reed M., Simon B., Methods of modern mathematical physics I, functional analysis, revised and enlarged edition, Academic Press, New York, 1980.

[3] Reed M., Simon B., Methods of modern mathematical physics II, Fourier analysis, self-adjointness, Academic Press, New York, 1975.

[4] Richtmyer R.D., Principles of advanced mathematical physics, Vol. I, Springer-Verlag, 1978.

[5] Akhiezer N.I., Glazman I.M., Theory of linear operators in Hilbert space, Pitman, New York, 1981.

[6] Gorbachuk V.I., Gorbachuk M.L., Boundary value problems for operator differential equations, Kluwer, 1991.

[7] Matolcsi T., Spacetime without reference frames, Akadémiai Kiadó, Budapest, 1993.

[8] Fülöp T., The physical role of boundary conditions in quantum mechanics, PhD dissertation, The University of Tokyo, Japan, 2006.

[9] Fülöp T., Cheon T., Tsutsui I., Classical aspects of quantum walls in one dimension, Phys. Rev. A 66 (2002), 052102-052111, quant-ph/0111057.

[10] Tsutsui I., Fülöp T., Cheon T., Duality and anholonomy in the quantum mechanics of 1D contact interactions, J. Phys. Soc. Japan 69 (2000), 3473-3476, quant-ph/0003069.

[11] Cheon T., Fülöp T., Tsutsui I., Symmetry, duality and anholonomy of point interactions in one dimension, Ann. Phys. (N.Y.) 294 (2001), 1-23, quant-ph/0008123.

[12] Tsutsui I., Fülöp T., Cheon T., Möbius structure of the spectral space of Schrödinger operators with point interaction, J. Math. Phys. 42 (2001), 5687-5697, quant-ph/0105066.

[13] Albeverio S., Gesztesy F., Høegh-Krohn R., Holden H., Solvable models in quantum mechanics, 2nd ed., with a remarkable Appendix written by P. Exner, AMS Chelsea Publishing, Providence, Rhode Island, 2005.

[14] Dittrich J., Exner P., Tunneling through a singular potential barrier, J. Math. Phys. 26 (1985), 2000-2008.

[15] Fehér L., Fülöp T., Tsutsui I., Inequivalent quantizations of the three-particle Calogero model constructed by separation of variables, Nuclear Phys. B 715 (2005), 713-757, math-ph/0412095.

[16] Cheon T., Tsutsui I., Fülöp T., Quantum abacus, Phys. Lett. A 330 (2004), 338-342, quant-ph/0404039.

[17] Gitman D., Tyutin I., Voronov B., Self-adjoint extensions as a quantization problem, Progr. Math. Phys., Birkhäuser, Basel, 2006.

[18] Frank W.M., Land D.J., Spector R.M., Singular potentials, Rev. Modern Phys. 43 (1971), 36-98.

[19] Krall A.M., Boundary values for an eigenvalue problem with a singular potential, J. Differential Equations 45 (1982), 128-138.

[20] Esteve J.G., Origin of the anomalies: The modified Heisenberg equation, Phys. Rev. D 66 (2002), 125013, 4 pages, hep-th/0207164.

[21] Coon S.A., Holstein B.R., Anomalies in quantum mechanics: the $1 / r^{2}$ potential, Amer. J. Phys. 70 (2002), 513-519, quant-ph/0202091.

[22] Essim A.M., Griffiths D.J., Quantum mechanics of the $1 / x^{2}$ potential, Amer. J. Phys. 74 (2006), 109-117. 